\title{
Flexible Multibody Dynamics and Sensitivity Analysis in the Design of a Morphing Leading Edge for High-Performance Sailplanes
}

\author{
Veit Gufler $^{1}$, Erich Wehrle ${ }^{1}$, Johannes Achleitner $^{1,2}$, Renato Vidoni $^{1}$ \\ ${ }^{1}$ Faculty of Science and Technology \\ Free University of Bozen-Bolzano \\ Universitätsplatz - Piazza Università 1 \\ 39100 Bozen-Bolzano, South Tyrol, Italy \\ ${ }^{2}$ Institute of Aircraft Design \\ Technical University of Munich \\ Boltzmannstr. 15 \\ 85748 Garching, Germany \\ \{Veit.Gufler, Erich.Wehrle, Renato.Vidoni\}@ unibz.it \\ Johannes.Achleitner@tum.de
}

\begin{abstract}
High-performance sailplanes have a wide speed range. Form-variable wings with a morphing leading edge in combination with a trailing flap for a high-speed and a lowspeed configuration have shown to increase the performance. In the present work, a morphing wing leading edge modeled as a system constituted of a flexible external wing skin and an actuation mechanism with rigid bodies is investigated. Flexible multibody dynamics with the floating frame of reference formulation is applied to analyze the behavior of the system during the motion from the high-speed configuration to the low-speed configuration. The methodology includes generalized- $\alpha$ time integration with Newton-Raphson iterations and efficient design sensitivity analysis. The design sensitivities are calculated with a semi-analytical approach based on direct differentiation. Of particular interest in the simulation are the deviations of the wing profile from the target shape in morphed configuration and the stresses in the external wing skin. The design variables used in the sensitivity analysis include geometric parameters, material parameters and loading parameters. The introduced methods show high efficiency and provides reliable results. The sensitivities are visualized giving an easy interpretation of the sensitivity values and in understanding how the design variables can be changed to improve the design. The sensitivity computations enable further investigations such as uncertainty analysis or gradient-based design optimization of the system.
\end{abstract}

Keywords: flexible multibody dynamics, sensitivity analysis, generalized- $\alpha$ method, morphing wing, morphing leading edge

\section{INTRODUCTION}

High-performance sailplanes have a large envelope of operating speeds. Fixed-geometry aircraft are designed to be a compromise for this wide speed range. Form-variable - or morphing - wings have shown to increase performance particularly when the leading edge of the wing is morphed in combination with a conventional trailing-edge flap [1,2,3]. Morphing leading edges have been modeled with traditional "hinged" mechanisms [2, 4] and with compliant mechanisms [3, 5]. Here, flexible multibody dynamics including rigid and flexible bodies is applied to a hinged mechanism. Flexible multibody simulation is a valuable tool to simulate and optimize flexible deformations and large displacements and rotations of such a mechanism. This enables the dynamic analysis during the motion of the mechanism in contrast to previous works in which the system was considered to be quasi-static $[2,3,4,5]$. In this work, we extend an in-house flexible multibody simulation code SIMULI to accommodate the simulation and sensitivity analysis of a morphing leading edge to be integrated in a future work in a design optimization framework.

The flexible multibody simulation approach of this work was developed and introduced in the application to cleaning mechanisms of Tyrolean weirs, intake systems of small Alpine hydroelectric plants $[6,7,8]$. The developed methodology includes efficient sensitivity analysis with the goal of 


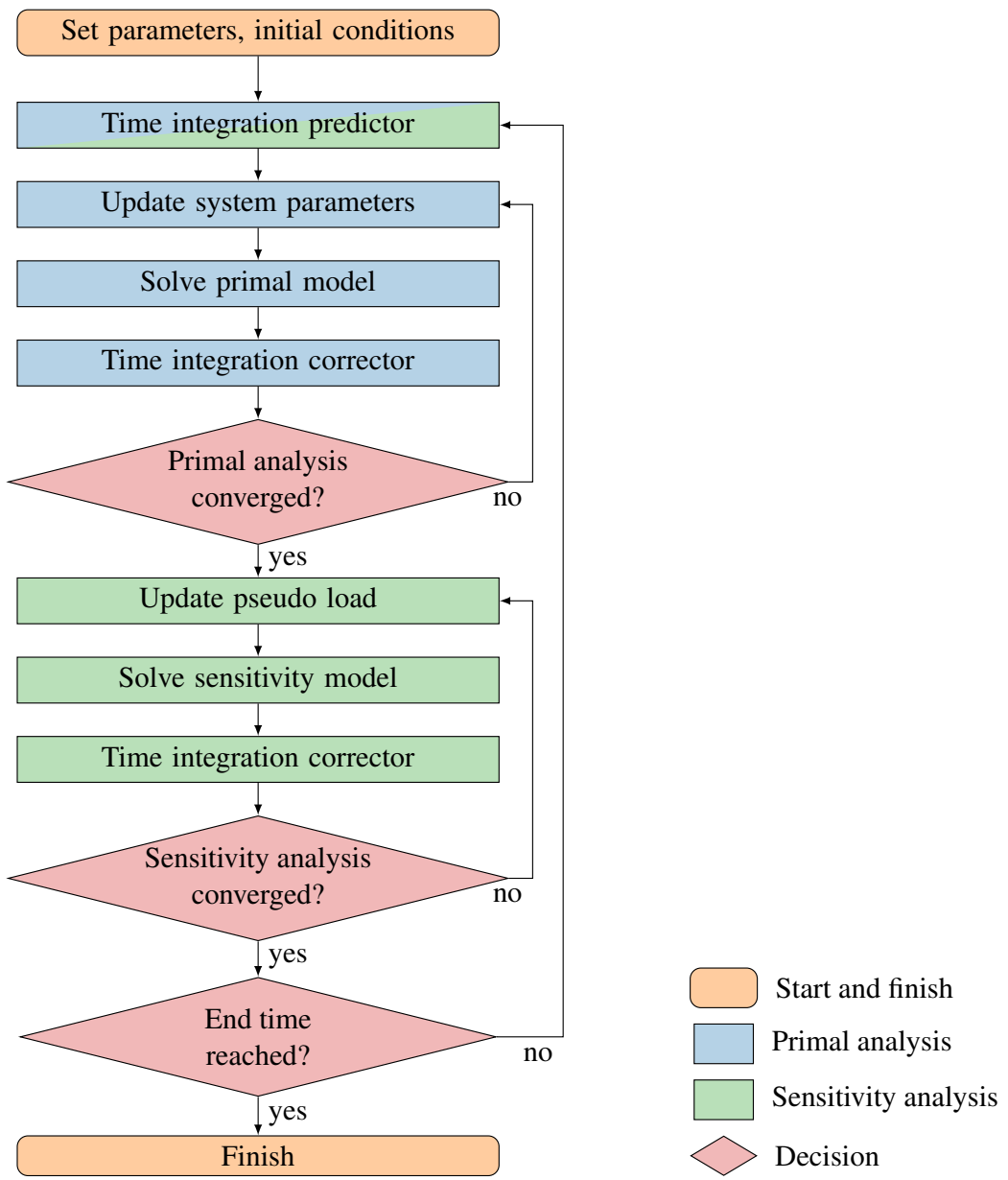

Figure 1: Flow chart for solving routine for flexible multibody dynamics and integrated sensitivity analysis

use in gradient-based design optimization of flexible mechanisms. This is developed for the simulation of a morphing leading edge concept using a flexible wing structure and a mechanism with rigid bodies driving the deformation from the high-speed profile configuration to the low-speed profile configuration. Special attention is paid to the computation, analysis and interpretation of design sensitivities. Therefore, the sensitivities of both stress and geometric deviation from the target geometry with respect to the design variables are investigated. The design variables considered here include geometric parameters, material parameters and loading parameters.

\section{FLEXIBLE MULTIBODY DYNAMICS}

The simulation of flexible multibody dynamics is categorized in three components as described in [8]: governing equation, time integration and nonlinear solver. The simulation is referred as primal analysis to differentiate from the sensitivity analysis. Fig. 1 shows the flow chart of the method and the components are introduced here in $\S 2$ for the primal analysis and in $\S 3$ for the sensitivity analysis.

\subsection{Governing equation}

The governing equations are given by index-1 differential-algebraic equations for the motion of flexible multibody systems and the constraint equations of kinematic joints,

$$
\underline{R}=\left[\begin{array}{cc}
\underline{\underline{m}} & \underline{\mathrm{J}}_{\Phi}^{T} \\
\underline{\underline{\mathrm{J}}} & \underline{\underline{0}}
\end{array}\right]\left[\begin{array}{c}
\ddot{q} \\
\underline{\lambda}
\end{array}\right]-\left[\begin{array}{c}
\underline{F}_{\mathrm{ext}}+\underline{F}_{v}-\underline{d} \underline{\dot{q}}-\underline{k} \underline{q} \underline{F_{c}} \\
\underline{F}^{\prime}
\end{array}\right]=\underline{0},
$$




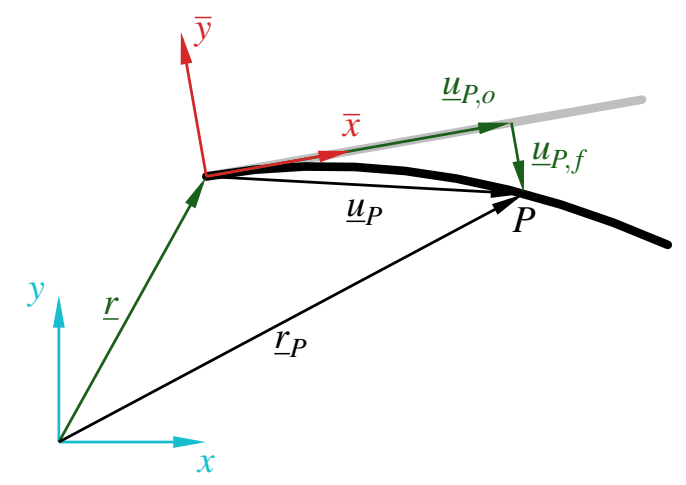

Figure 2: Floating frame of reference formulation (FFRF)

where $R$ is the residual, $q$ is the generalized position, $\lambda$ are Lagrangian multipliers of the kinematic constraints, $m$ is the mass, $d$ is the damping, $k$ is the stiffness, $\Phi$ are kinematic constraints, $\mathrm{J}$ is the Jacobian (i.e. the partial derivative with respect to position), $F_{\text {ext }}$ is the external force, $F_{c}$ is the right hand side of acceleration constraints, $F_{v}$ is the quadratic velocity force and overdots represent the first $\star$ and second $\ddot{\star}$ differentiation with respect to time. Single underlined symbols $₫$ represent vectors, double underlined symbols $\underline{\underline{ }}$ are two-dimensional matrices, triple underlined symbols are three-dimensional and quadruple underlined symbols are four-dimensional, those without under-

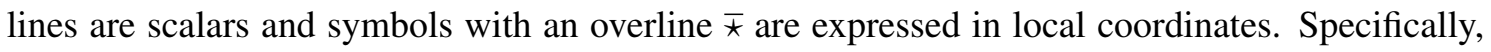
the floating frame of reference formulation (FFRF) is used to represent flexibility [9, 10]. The generalized positions of a flexible body with FFRF is given by the position $\underline{r}$ and orientation $\underline{\theta}$ of the reference frame and flexible deformations $\underline{\bar{q}}_{f}$, see fig. 2,

$$
\underline{q}=\left[\begin{array}{lll}
\underline{r}^{T} & \underline{\theta}^{T} & \bar{q}_{f}^{T}
\end{array}\right]^{T} .
$$

The continuous position of a material point on a flexible body is

$$
\underline{\underline{r}}_{P}=\underline{r}+\underline{\underline{A}} \overline{\underline{u}}_{P}=\underline{r}+\underline{\underline{A}}\left(\underline{\bar{u}}_{P, o}+\underline{\underline{S}}_{\underline{\underline{q}}}\right),
$$

where $\underline{\underline{A}}$ is the transformation matrix and $\underline{\underline{u}}_{P}$ is the local position vector decomposed by the undeformed term $\underline{\underline{u}}_{P, o}$ and the deformed term $\underline{\underline{u}}_{P, f}$ given by the local matrix of shape functions $\underline{\underline{S}}$ and the nodal deformations $\underline{\underline{q}}_{f}$. In this implementation of FFRF, a linear-elastic material model is used, which leads to a linear stiffness matrix,

$$
\underline{\underline{k}}=\left[\begin{array}{ccc}
\underline{\underline{0}} & \underline{\overline{0}} & \underline{\overline{0}} \\
\underline{\overline{0}} & \underline{\overline{0}} & \underline{\underline{0}} \\
\underline{\underline{0}} & \underline{\underline{0}} & \underline{\underline{k}} f f
\end{array}\right],
$$

with the finite-element stiffness matrix $\underline{\underline{k}}_{f f}$. In contrast, the mass matrix is highly nonlinear

$$
\begin{aligned}
& \underline{\underline{m}}=\left[\begin{array}{ccc}
\underline{\underline{m}}_{t} & \underline{\underline{m}}_{t r} & \underline{\underline{m}}_{t} \\
& \underline{\underline{m}}_{r r} & \underline{\underline{m}}_{f f} \\
\text { sym. } & & \underline{\underline{B}}_{f}
\end{array}\right],
\end{aligned}
$$

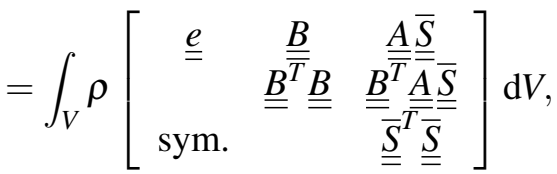

with the finite-element mass matrix $\underline{\underline{m}}_{f f}$, the volume $V$, the density $\rho$, the identity matrix $\underline{\underline{e}}$ and the matrix $\underline{\underline{B}}$. The lattermost term is defined by

$$
\underline{\underline{B}}=-\underline{\underline{A}} \underline{\underline{\underline{u}}}_{P} \underline{\underline{\bar{G}}}
$$


with the skew-symmetric matrix of local coordinates $\underline{\underline{\bar{u}}}_{P}$ and the matrix $\underline{\underline{\underline{G}}}$ that relates the local angular velocity vector $\underline{\overline{\underline{ }}}$ and the velocity terms of the orientation parameters $\underline{\dot{\beta}}$,

$$
\underline{\bar{\omega}}=\underline{\underline{G}} \underline{\dot{\beta}} .
$$

Moreover, the quadratic velocity vector is also highly nonlinear

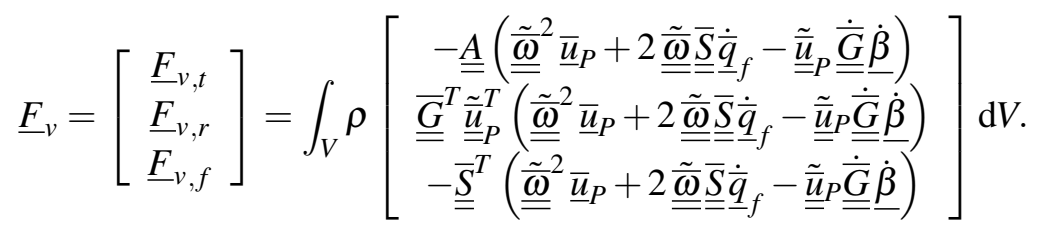

The vector of external forces in FFRF is

$$
\underline{F}_{\mathrm{ext}}=\left[\begin{array}{c}
\underline{\underline{e}} \\
\underline{\underline{\underline{B}}}^{T} \\
\underline{\underline{T}}^{\underline{\underline{A}}}{ }^{T}
\end{array}\right] \underline{F}_{\mathrm{ext}} .
$$

The introduced governing equation must be fulfilled at any time and needs to be solved for every time step of the flexible multibody simulation.

\subsection{Time integration}

Numerical time integration is carried out with the generalized- $\alpha$ method originally introduced by [11] and implemented as a predictor-corrector scheme, as described in [8, 12]. The method is based on Newmark's equations,

$$
\begin{aligned}
& \underline{q}_{n+1}=\underbrace{\underline{q}_{n}+\Delta t \underline{\dot{q}}_{n}+\left(\frac{1}{2}-\beta\right) \Delta t^{2} \underline{\underline{q}}_{n}}_{\text {predictor } \underline{q}_{\text {pred }}}+\underbrace{\beta \Delta t^{2} \underline{\underline{q}}_{n+1}}_{\text {corrector }}, \\
& \underline{\dot{q}}_{n+1}=\underbrace{\dot{\dot{q}}_{n}+(1-\gamma) \Delta t \underline{\ddot{q}}_{n}}_{\text {predictor } \dot{\underline{q}}_{\text {pred }}}+\underbrace{\gamma \Delta t \underline{\ddot{q}}_{n+1}}_{\text {corrector }},
\end{aligned}
$$

and intermediate approximations for all force terms,

$$
\begin{aligned}
& \underline{\underline{m}} \underline{\ddot{q}}=\left(1-\alpha_{m}\right) \underline{\underline{m}}_{n+1} \underline{\ddot{q}}_{n+1}+\alpha_{m} \underline{\underline{m}}_{n} \underline{\ddot{q}}_{n}, \\
& \underline{\underline{d}} \underline{\dot{q}}=\left(1-\alpha_{f}\right) \underline{\underline{d}}_{n+1} \underline{\dot{q}}_{n+1}+\alpha_{f} \underline{\underline{d}}_{n} \underline{\dot{q}}_{n}, \\
& \underline{\underline{k}} \underline{q}=\left(1-\alpha_{f}\right) \underline{\underline{k}}_{n+1} \underline{q}_{n+1}+\alpha_{f} \underline{\underline{k}}_{n} \underline{q}_{n}, \\
& \underline{\underline{\mathbf{J}}}_{\Phi}^{T} \underline{\boldsymbol{\lambda}}=\left(1-\alpha_{m}\right) \underline{\underline{\mathbf{J}}}_{\Phi, n+1}^{T} \underline{\lambda}_{n+1}+\alpha_{m} \underline{\underline{\mathbf{J}}}_{\Phi, n}^{T} \underline{\boldsymbol{\lambda}}_{n}, \\
& \underline{\mathrm{J}}_{\Phi} \underline{\ddot{q}}=\left(1-\alpha_{m}\right) \underline{\mathrm{J}}_{\Phi, n+1} \underline{\ddot{q}}_{n+1}+\alpha_{m} \underline{\underline{\mathrm{J}}}_{\Phi, n} \underline{\ddot{q}}_{n}, \\
& \underline{F}_{\mathrm{ext}}=\left(1-\alpha_{f}\right) \underline{F}_{\mathrm{ext}, n+1}+\alpha_{f} \underline{F}_{\mathrm{ext}, n}, \\
& \underline{F}_{v}=\left(1-\alpha_{f}\right) \underline{F}_{v, n+1}+\alpha_{f} \underline{F}_{v, n}, \\
& \underline{F}_{c}=\left(1-\alpha_{f}\right) \underline{F}_{c, n+1}+\alpha_{f} \underline{F}_{c, n} .
\end{aligned}
$$

These lead to the effective system of equations of flexible multibody dynamics,

$$
\left[\begin{array}{cc}
\underline{\underline{m}}_{\mathrm{eff}} \mathrm{eff} & \underline{\mathbf{J}}_{\Phi, \mathrm{eff}}^{T} \\
\underline{\underline{\mathbf{J}}}_{\Phi, \mathrm{eff}} & \underline{\underline{0}}
\end{array}\right]\left[\begin{array}{c}
\ddot{q}_{n+1} \\
\underline{\boldsymbol{\lambda}}_{n+1}
\end{array}\right]=\left[\begin{array}{l}
\underline{F}_{a, \mathrm{eff}} \\
\underline{F}_{c, \mathrm{eff}}
\end{array}\right],
$$


where

$$
\begin{aligned}
\underline{\underline{m}}_{\mathrm{eff}}= & \left(1-\alpha_{m}\right) \underline{\underline{m}}_{n+1}+\left(1-\alpha_{f}\right) \gamma \Delta t \underline{\underline{d}}_{n+1}+\left(1-\alpha_{f}\right) \beta \Delta t^{2} \underline{k}_{n+1}, \\
\underline{\underline{\mathrm{J}}}_{\Phi, \mathrm{eff}}= & \left(1-\alpha_{m}\right) \underline{\mathrm{J}}_{\Phi, n+1}, \\
\underline{F}_{a, \mathrm{eff}}= & \left(1-\alpha_{f}\right) \underline{F}_{\mathrm{ext}, n+1}+\alpha_{f} \underline{F}_{\mathrm{ext}, n}+\left(1-\alpha_{f}\right) \underline{F}_{v, n+1}+\alpha_{f} \underline{F}_{v, n}-\alpha_{m} \underline{\underline{m}}_{n} \underline{\ddot{q}}_{n}+ \\
& -\left(1-\alpha_{f}\right) \underline{\underline{d}}_{n+1} \underline{\dot{q}}_{\mathrm{pred}}-\alpha_{f} \underline{\underline{d}}_{n} \underline{\dot{q}}_{n}-\left(1-\alpha_{f}\right) \underline{\underline{k}}_{n+1} \underline{q}_{\mathrm{pred}}-\alpha_{f} \underline{\underline{k}}_{n} \underline{q}_{n}-\alpha_{m} \underline{\mathrm{J}}_{\Phi, n}^{T} \underline{\lambda}_{n}, \\
\underline{F}_{c, \mathrm{eff}}= & \left(1-\alpha_{f}\right) \underline{\underline{F}}_{c, n+1}+\alpha_{f} \underline{\underline{F}}_{c, n}-\alpha_{m} \underline{\mathrm{J}}_{\Phi, n} \underline{\ddot{q}}_{n} .
\end{aligned}
$$

With the new acceleration values, the updates for the position and velocity values can be performed and the simulation continues to the next time step.

\subsection{Nonlinear solver}

With generalized- $\alpha$ time integration, the residual equations are

$$
\begin{aligned}
\underline{R}_{1, n+1-\alpha_{f}}= & \left(1-\alpha_{m}\right) \underline{\underline{m}}_{n+1} \underline{\underline{q}}_{n+1}+\alpha_{m} \underline{\underline{m}}_{n} \underline{\underline{q}}_{n}+\left(1-\alpha_{f}\right) \underline{\underline{d}}_{n+1} \underline{\dot{q}}_{n+1}+\alpha_{f} \underline{\underline{d}}_{n} \underline{\dot{q}}_{n}+ \\
& +\left(1-\alpha_{f}\right) \underline{k}_{n+1} \underline{q}_{n+1}+\alpha_{f} \underline{k}_{n} \underline{q}_{n}+\left(1-\alpha_{m}\right) \underline{\underline{\mathbf{J}}}_{\Phi, n+1}^{T} \underline{\lambda}_{n+1}+\alpha_{m} \underline{\mathrm{J}}_{\Phi, n}^{T} \underline{\lambda}_{n}+ \\
& -\left(1-\alpha_{f}\right) \underline{\boldsymbol{F}}_{\mathrm{ext}, n+1}-\alpha_{f} \underline{\mathrm{F}}_{\mathrm{ext}, n}-\left(1-\alpha_{f}\right) \underline{F}_{v, n+1}-\alpha_{f} \underline{F}_{v, n}, \\
\underline{R}_{2, n+1-\alpha_{f}}= & \left(1-\alpha_{m}\right) \underline{\underline{\mathrm{J}}}_{\Phi, n+1} \underline{\ddot{q}}_{n+1}+\alpha_{m} \underline{\mathrm{J}}_{\Phi, n} \underline{\ddot{q}}_{n}-\left(1-\alpha_{f}\right) \underline{F}_{c, n+1}-\alpha_{f} \underline{F}_{c, n} .
\end{aligned}
$$

To consider the nonlinearities of the system, Newton-Raphson iterations are used here with the following equation

$$
\left[\begin{array}{ll}
\ddot{\mathrm{J}}_{R} & \lambda \\
\underline{\mathbf{J}}_{R}
\end{array}\right]\left[\begin{array}{l}
\Delta \ddot{\ddot{q}} \\
\Delta \underline{\hat{\lambda}}
\end{array}\right]+\underline{R}=\underline{0}
$$

where $\ddot{J}$ and $\stackrel{\lambda}{J}$ are the Jacobians with respect to acceleration and Lagrangian multipliers,

$$
\begin{aligned}
& \ddot{\underline{\mathrm{J}}}_{R}=\frac{\partial \underline{R}}{\partial \underline{\ddot{q}}}, \\
& \stackrel{\mathrm{J}}{=}_{R}=\frac{\partial \underline{R}}{\partial \underline{\lambda}} .
\end{aligned}
$$

The terms of the Jacobian matrix are

$$
\begin{aligned}
& \underline{\underline{\mathrm{J}}}_{R_{1}}=\left(1-\alpha_{m}\right) \beta \Delta t^{2} \underline{\underline{J}}_{m, n+1} \underline{\ddot{q}}_{n+1}+\left(1-\alpha_{m}\right) \underline{\underline{m}}_{n+1}+ \\
& +\left(1-\alpha_{f}\right) \beta \Delta t^{2} \underline{\underline{J}}_{d, n+1} \underline{\dot{q}}_{n+1}+\left(1-\alpha_{f}\right) \gamma \Delta t \underline{\underline{d}}_{n+1}+ \\
& +\left(1-\alpha_{f}\right) \beta \Delta t^{2} \underline{\underline{J}}_{k, n+1} \underline{q}_{n+1}+\left(1-\alpha_{f}\right) \beta \Delta t^{2} \underline{\underline{k}}_{n+1}+ \\
& +\left(1-\alpha_{m}\right) \beta \Delta t^{2} \underline{\Xi}_{\Phi, n+1}^{T} \underline{\lambda}_{n+1}+ \\
& -\left(1-\alpha_{f}\right)\left(\beta \Delta t^{2} \underline{\underline{J}}_{F_{\text {ext }}, n+1}+\gamma \Delta t \underline{\underline{\mathbf{J}}}_{F_{\text {ext }}, n+1}\right)+ \\
& -\left(1-\alpha_{f}\right)\left(\beta \Delta t^{2} \underline{\underline{\mathbf{J}}}_{F_{v}, n+1}+\gamma \Delta t \underline{\underline{\mathbf{J}}}_{F_{v}, n+1}\right), \\
& \underline{\underline{\mathbf{J}}}_{R_{2}}=\left(1-\alpha_{m}\right) \beta \Delta t^{2} \underline{\underline{J}}_{\Phi, n+1}^{T} \underline{\ddot{q}}_{n+1}+\left(1-\alpha_{m}\right) \underline{\underline{\mathbf{J}}}_{\Phi, n}+ \\
& -\left(1-\alpha_{f}\right)\left(\beta \Delta t^{2} \underline{\underline{\mathbf{J}}} F_{c}, n+1+\gamma \Delta t \underline{\underline{\mathbf{J}}} F_{c}, n+1\right), \\
& {\stackrel{\underline{\mathrm{J}}}{R_{1}}}_{R_{1}}=\left(1-\alpha_{m}\right) \underline{\mathrm{J}}_{\Phi, n+1}^{T}, \\
& \underline{\underline{\mathrm{J}}} R_{2}=\underline{\underline{0}} \text {. }
\end{aligned}
$$

A complete derivation of the terms for the effective system and the nonlinear solver including the design sensitivities is found in [12]. 


\section{DESIGN SENSITIVITY ANALYSIS}

The sensitivities of the system responses with respect to certain parameters are useful in design optimization, uncertainty analysis as well as the direct use of the sensitivities. In this work, the design sensitivity analysis is carried out with a semi-analytical approach using direct differentiation. The differentiation must be carried through all three steps of the calculation routine: governing equation, sensitivity analysis and nonlinear solver.

\subsection{Governing equation}

The direct differentiation of the primal equations results in the governing equations for the sensitivity analysis,

$$
\underline{\underline{\nabla R}}=\left[\begin{array}{cc}
\underline{\underline{m}} & \underline{\underline{\mathrm{J}}}_{\Phi}^{T} \\
\underline{\underline{\mathrm{J}_{\Phi}}} & \underline{\underline{0}}
\end{array}\right]\left[\begin{array}{c}
\underline{\nabla \ddot{q}} \\
\underline{\underline{\nabla \lambda}}
\end{array}\right]-\underline{\underline{F}}_{\mathrm{pseudo}}
$$

where the pseudo load $\underline{\underline{F}}_{\text {pseudo }}$ contains the partial derivatives of the system parameters with respect to the design variables,

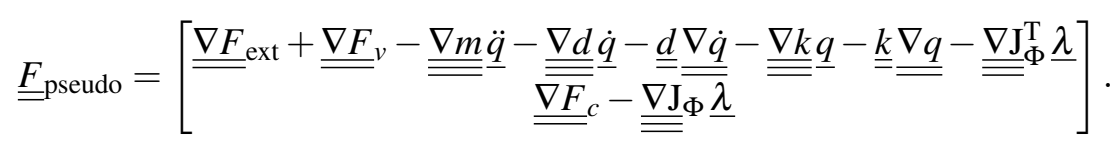

To limit the implementation effort in the simulation code, the partial derivatives are evaluated with numerical forward differences, thus resulting in a semi-analytical approach.

\subsection{Time integration}

The time integration for the sensitivities is analogous to the time integration of the primary analysis as a predictor-corrector scheme with Newmark's equations and intermediate approximations $[8$, 12]. Therefore, the effective system of equations of the sensitivity analysis is

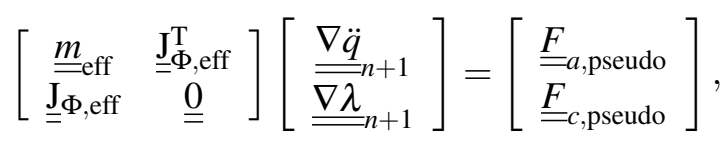

where the pseudo load case is

$$
\begin{aligned}
& \underline{\underline{F}}_{a, \text { pseudo }}=\left(1-\alpha_{f}\right) \underline{\underline{\nabla F}}_{\mathrm{ext}, n+1}+\alpha_{f} \underline{\underline{\nabla F}}_{\mathrm{ext}, n}+\left(1-\alpha_{f}\right) \underline{\underline{\nabla F}}_{v, n+1}+\alpha_{f} \underline{\underline{\nabla F}}_{v, n}+ \\
& -\left(1-\alpha_{m}\right) \underline{\underline{\underline{\nabla m}}}_{n+1} \underline{\ddot{q}}_{n+1}-\alpha_{m} \underline{\underline{\underline{\nabla m}}}_{n} \underline{\ddot{q}}_{n}-\alpha_{m} \underline{\underline{m}}_{n} \underline{\underline{\underline{q}}}_{n}+ \\
& -\left(1-\alpha_{f}\right) \underline{\underline{\underline{\nabla d}}}_{n+1} \underline{\dot{q}}_{n+1}-\alpha_{f} \underline{\underline{\underline{\nabla d}}}_{n} \dot{q}_{n}-\left(1-\alpha_{f}\right) \underline{\underline{d}}_{n+1} \underline{\underline{\nabla}}_{\text {pred }}-\alpha_{f} \underline{\underline{d}}_{n} \underline{\underline{\dot{q}_{q}}} n \\
& -\left(1-\alpha_{f}\right) \underline{\underline{\underline{\nabla k}}}_{n+1} \underline{q}_{n+1}-\alpha_{f} \underline{\underline{\underline{\nabla k}}}_{n} \underline{q}_{n}-\left(1-\alpha_{f}\right) \underline{\underline{k}}_{n+1} \underline{\underline{\nabla}}_{\text {pred }}-\alpha_{f} \underline{\underline{k}}_{n} \underline{\underline{\nabla q}}_{n}+ \\
& -\left(1-\alpha_{m}\right) \underline{\underline{\underline{\mathrm{J}}}}_{\Phi, n+1}^{T} \underline{\lambda}_{n+1}-\alpha_{m} \underline{\underline{\underline{\mathrm{J}}}}_{\Phi, n}^{T} \underline{\lambda}_{n}-\alpha_{m} \underline{\underline{\mathrm{J}}}_{\Phi, n}^{T} \underline{\underline{\nabla \lambda}}_{n}, \\
& \underline{\underline{F}}_{c, \text { pseudo }}=\left(1-\alpha_{f}\right) \underline{\underline{\nabla F}}_{c, n+1}+\alpha_{f} \underline{\underline{\nabla F}}_{c, n}-\left(1-\alpha_{m}\right) \underline{\underline{\underline{\nabla}}}_{\Phi, n+1} \underline{\ddot{q}}_{n+1}-\alpha_{m} \underline{\underline{\underline{\nabla \mathrm{J}}}}_{\Phi, n} \underline{\ddot{q}}_{n}-\alpha_{m} \underline{\underline{\mathrm{J}}}_{\Phi, n} \underline{\underline{\nabla}} \underline{\underline{q}} .
\end{aligned}
$$

The updates from the acceleration values to the position and velocity values are performed analogously to the primal analysis.

\subsection{Nonlinear solver}

The Jacobian of the sensitivity analysis is that of the primary analysis $[8,12]$, allowing for an efficient calculation method of the gradients,

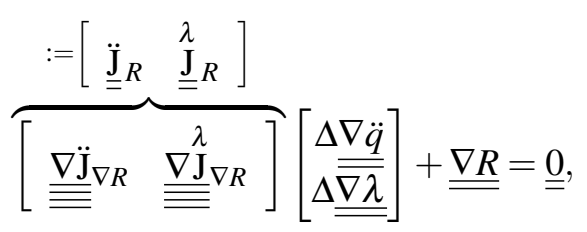




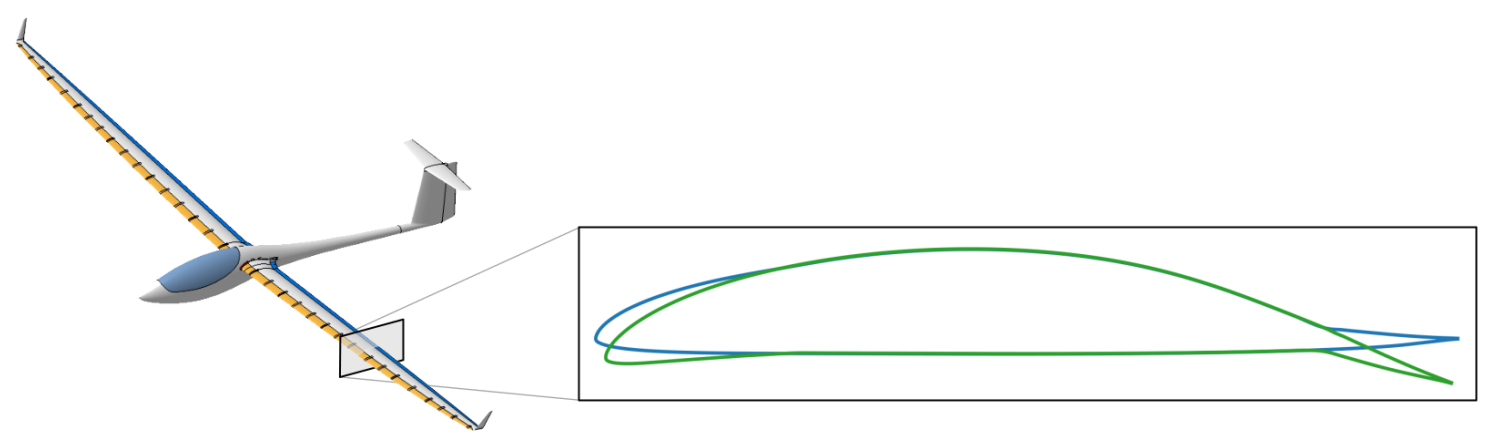

Figure 3: Demonstration example for a morphing wing with airfoil shapes for — high-speed and - low-speed configurations.

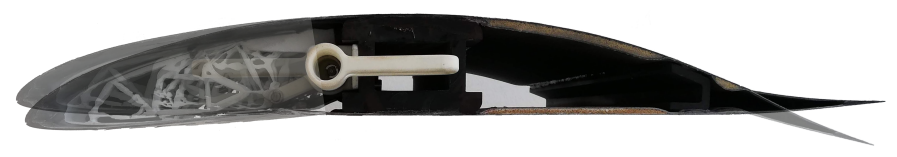

Figure 4: Morphing wing demonstrator based on topology optimization results in undeformed and morphed configuration [5]

where $\nabla \ddot{\mathrm{J}}$ and $\nabla \stackrel{\lambda}{\mathrm{J}}$ are the Jacobians with respect to acceleration sensitivity and Lagrangian multipliers sensitivity, respectively. This is the key to efficient sensitivity analysis of multibody dynamics. Without this simplification, the Jacobian of the sensitivity analysis is four-dimensional, requiring excessive memory usage. This is avoided by recognizing that these Jacobians are equivalent to those from the primary analysis and therefore avoiding any further calculation.

\section{MORPHING WING MODEL}

The design of the target geometry of the morphing wing sailplane in undeformed and morphed configuration is presented in $[1,13]$ and shown in fig. 3. Airfoil shapes are designed using a numerical optimization approach considering aerodynamic lift and drag $[14,15]$. A morphing concept shows a significant performance advantage over conventional sailplanes with a camber changing flap. In earlier work [5], the morphing actuation is achieved using compliant mechanisms. A concept with a stack of six individual compliant mechanisms is shown in fig. 4.

Flexible multibody dynamics with FFRF is applied to the planar model of the system shown in fig. 5a. A conventional hinged mechanism is investigated for actuation. The flexible outer shell, i.e. wing skin, (body 1) is modeled with planar Euler-Bernoulli beams considering a section of the wing profile with the length of $1000 \mathrm{~mm}$ and a wall thickness of $2 \mathrm{~mm}$. The mesh consists of 31 nodes and 30 elements. The upper end of the leading edge wing profile (point $F$ ) is fixed in all three degrees of freedom, i.e. $x, y$ and $\theta$. The lower end of the leading edge wing profile (point $\mathrm{G}$ ) is fixed in $y$ and $\theta$, allowing for free motion in $x$. The actuation mechanism consists of four rigid bodies 2, 3, 4 and 5. These are connected by five revolute joints in A, B, C, D and E and a rigid joint between the bodies 2 and 4 in $\mathrm{B}$. The mechanism is actuated by a torque starting from zero and going to a maximum value of $10 \mathrm{Nm}$ that is applied to body 2 on point A. Aluminum is used as material with a density of $2.7 \times 10^{-9} \mathrm{t} / \mathrm{mm}^{3}$, a Young modulus of $70000 \mathrm{MPa}$ and a Poisson ratio of 0.35 [-]. Fig. 5b shows the motion of the multibody system from the high-speed configuration (undeformed) to the low-speed configuration (morphed).

The key element for a proper function of the morphing leading edge is the design of the actuation mechanism considering the interaction with the flexible wing skin. It is important to approximate the target wing profile in morphed configuration as closely as possible. Another important aspect is to consider the material limits. A simulation with flexible multibody dynamics is performed 


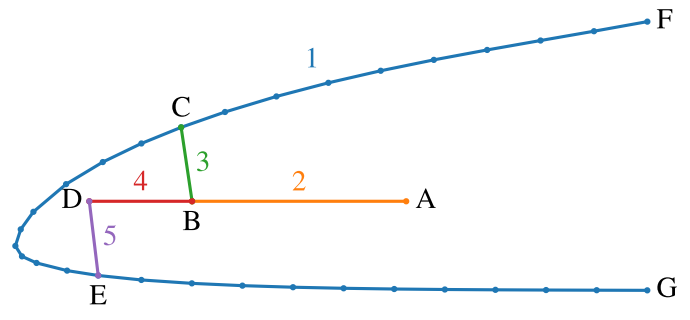

(a) Model of mechanism and wing skin

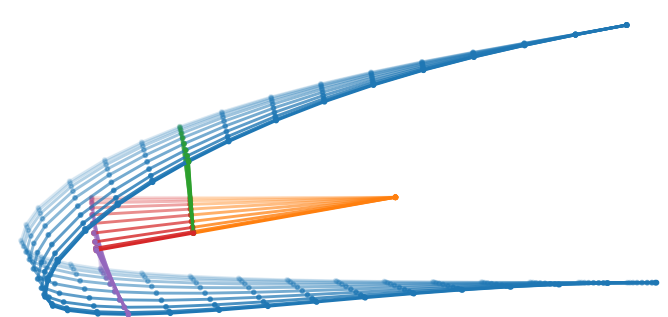

(b) Motion of rigid mechanism and deformable wing skin

Figure 5: Flexible multibody system of the morphing wing leading edge

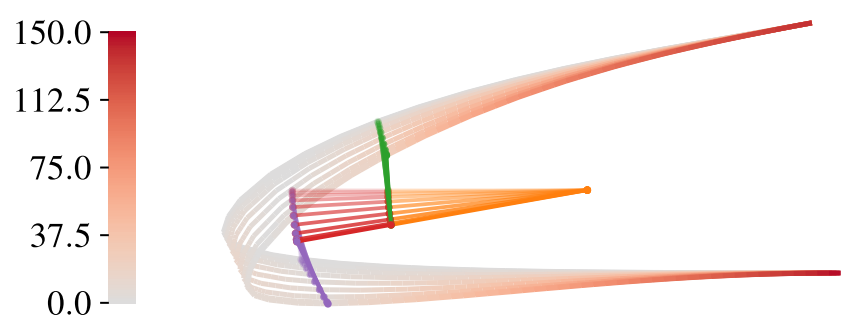

Figure 6: Stress $\sigma[\mathrm{MPa}]$ during the motion of the mechanism

to assess both deformed shape as well as the stresses. Fig. 6 shows the stress distribution in the wing skin during the motion of the mechanism from the undeformed configuration to the morphed configuration. The highest values are found in morphed configuration and near to the constraints of the wing skin (points $F$ and $G$ ). The maximum stress of all elements and all time steps is approximated with the Kreisselmeier-Steinhauser function [16] to obtain a differentiable function

$$
\sigma_{\max }=\mathscr{F}_{K S}\left(\sigma^{i, j}\right), \quad i=1, \ldots, n_{t}, j=1, \ldots, n_{e},
$$

where $\mathscr{F}_{K S}$ is the Kreisselmeier-Steinhauser function, $\sigma$ is the stress, $n_{t}$ is the number of time steps and $n_{e}$ is the number of elements. Here, the approximated maximum stress is given by 149.96 MPa.

Fig. 7a shows the external wing profile in the undeformed configuration, the target morphed configuration and the simulated morphed configuration. It is possible to observe an error between the simulated morphed configuration and the target morphed configuration. This error is given by the distance between the simulated node positions and the target profile in morphed configuration,

$$
e_{k}=\sqrt{\left(x_{s}^{k}-x_{t}^{k}\right)^{2}+\left(y_{s}^{k}-y_{t}^{k}\right)^{2}}, \quad k=1, \ldots, n_{N},
$$

where $x_{s}^{k}$ and $y_{s}^{k}$ are the simulated node coordinates, $x_{t}^{k}$ and $y_{t}^{k}$ are the target node coordinates and $n_{N}$ is the number of nodes. Fig. $7 \mathrm{~b}$ shows the error value of the morphed configuration. The largest error is $1.185 \mathrm{~mm}$ and is located behind the tip on the lower side of the wing profile.

The deviation from the target shape as a global consideration is expressed via root-mean-square error,

$$
e_{\mathrm{rms}}=\sqrt{\frac{1}{n_{N}} \sum_{k=1}^{n_{N}} e_{k}^{2}},
$$

and is given by $0.6348 \mathrm{~mm}$. The deviations from the target wing profile with a maximum value of $1.185 \mathrm{~mm}$ and a root-mean-square error of $0.6348 \mathrm{~mm}$ are to acceptable accuracy, especially considering the wing width of $550 \mathrm{~mm}$, the wing height of $67.5 \mathrm{~mm}$ and the width of the leading edge of $137.5 \mathrm{~mm}$. Nevertheless, it is important to analyze and minimize the deviations, since the shape of the wing profile is crucial for the aerodynamics and the performance of sailplanes. 


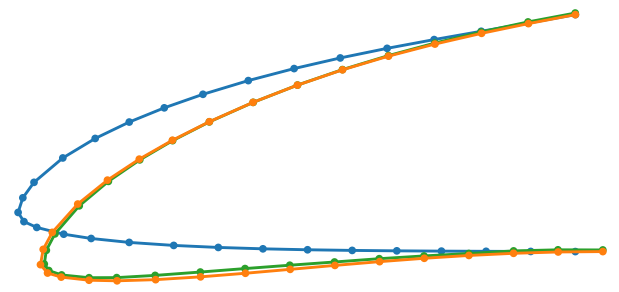

(a) - undeformed configuration - target configuration - simulated configuration

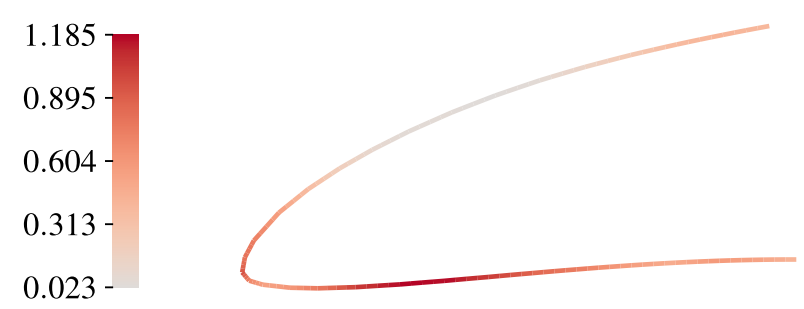

(b) Error in morphed configuration $e[\mathrm{~mm}]$

Figure 7: Shape of the external wing profile

To understand how the stress distribution and the deviation from the target profile are affected by the design variables, a sensitivity analysis is performed. The sensitivity analysis is performed with respect to the design variables, which include the thickness of the external wing skin $t_{w}$, the value of the torque that is applied to actuate the mechanism $M_{t}$, the coordinates $x_{A}$ and $y_{A}$ of the point where the torque is applied (point A) and the Young's modulus $E$. The sensitivities are computed as shown in $\S 3$ and the chain rule is applied to all operations.

Fig. 8 shows the stress sensitivities during the motion of the system ${ }^{1}$. In terms of simulation time, the highest sensitivity values are always found at the end of the motion in morphed configuration of the wing profile. In terms of position on the wing profile, the highest sensitivity values with respect to the $x$-coordinate $x_{A}$ of the application point of the torque are found on the tip of the wing profile and on the upper connection point between the actuation mechanism and the external wing profile (point $\mathrm{C}$ ), while for all other design variables, the highest sensitivity values are found near to the constraints of the wing profile (points F and G). In contrast to the primal analysis, where the highest stress values are found on the lower constraint (point $G$ ), the highest values of the stress sensitivities are on the upper constraint (point $F$ ). A comparison between fig. 6 and fig. 8 allows to conclude that the stress $\sigma$ can be reduced by increasing the thickness of the external wing skin $t_{w}$, the $x$-coordinate $x_{A}$ of the application point of the torque and the Young's modulus $E$ or by reducing the actuation torque $M_{t}$ and the $y$-coordinate $y_{A}$ of the application point of the torque.

Fig. 8 shows the sensitivities of the error in morphed configuration. Here it is possible to observe that especially in the nose of the leading edge, the sensitivities on the lower side have the opposite sign from the sensitivities on the upper side of the wing profile. Comparing the error values on fig. $7 \mathrm{~b}$ with the error sensitivities on fig. 9 allows to predict in which direction the design variables should be modified in order to reduce the error. Since the highest values of the error are on the lower side of the wing skin, these can be reduced by increasing the thickness of the external wing skin $t_{w}$, the $x$-coordinate $x_{A}$ of the application point of the torque and the Young's modulus $E$ or by reducing the actuation torque $M_{t}$ and the $y$-coordinate $y_{A}$ of the application point of the torque.

Tab. 1 gives the sensitivity values of the maximum stress and the root-mean-square error. These have been computed by the differentiation of the Kreisselmeier-Steinhauser function and the function of the root-mean-square error. Comparing the values from tab. 1 with the values on fig. 8 and fig. 9, the results show great consistency. In the case of the maximum stress, the maximum value is found on the lower end of the wing profile leading edge (point G). The sensitivity on this position of fig. 8 corresponds exactly to the approximated values reported in tab. 1 . The same comparison is not possible for the root-mean-square error because all nodes are considered by the function, but the values from fig. 9 and tab. 1 show the same order of magnitude.

The results from the semi-analytical sensitivity analysis are validated with numerical sensitivity analysis and the values coincide. Table 2 shows the computation time for both methods ${ }^{2}$. This

\footnotetext{
${ }^{1}$ Units of sensitivity results are not reduced to demonstrate the physical and engineering meaning of these values.

${ }^{2}$ Computations are performed on a PC with Intel Core i7-8700 CPU @ 3.20GHz $\times 12$ and 32 GB RAM.
} 

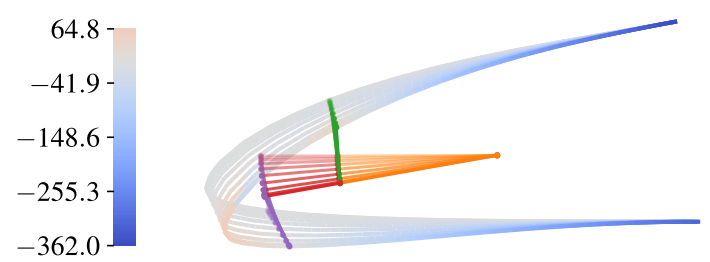

(a) Sensitivity w.r.t. thickness $\frac{\partial \sigma}{\partial t_{w}}[\mathrm{MPa} / \mathrm{mm}]$

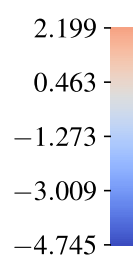

(c) Sensitivity w.r.t. actuation point in $x \frac{\partial \sigma}{\partial x_{a}}[\mathrm{MPa} / \mathrm{mm}]$
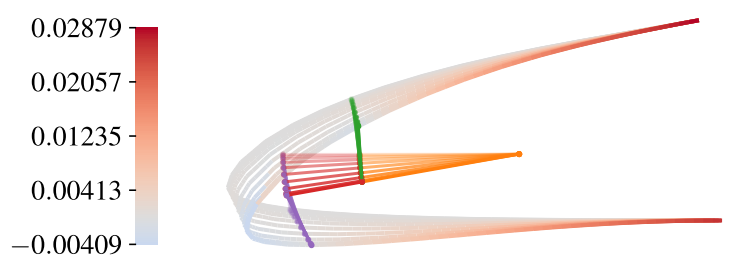

(b) Sensitivity w.r.t. torque $\frac{\partial \sigma}{\partial M_{t}}[\mathrm{MPa} / \mathrm{N} \cdot \mathrm{mm}]$
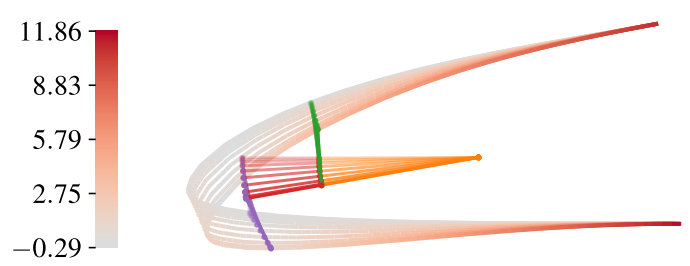

(d) Sensitivity w.r.t. actuation point in $y \frac{\partial \sigma}{\partial y_{a}}[\mathrm{MPa} / \mathrm{mm}]$

$$
\begin{array}{r}
0.000683- \\
-0.000027- \\
-0.000738- \\
-0.001448- \\
-0.002158
\end{array}
$$

(e) Sensitivity w.r.t. Young's modulus $\frac{\partial \sigma}{\partial E}[\mathrm{MPa} / \mathrm{MPa}]$

Figure 8: Design sensitivities of the stress $\sigma$
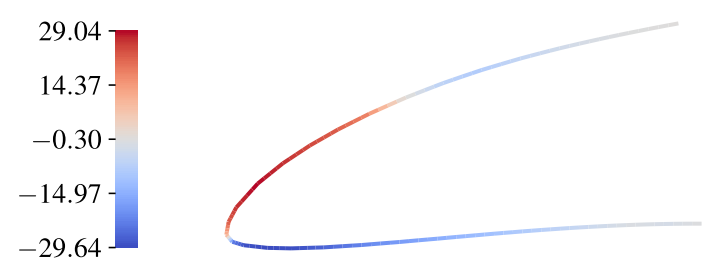

(a) Sensitivity w.r.t. thickness $\frac{\partial e}{\partial t_{w}}[\mathrm{~mm} / \mathrm{mm}]$

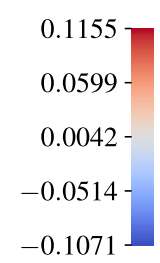

(c) Sensitivity w.r.t. actuation point in $x \frac{\partial e}{\partial x_{a}}[\mathrm{~mm} / \mathrm{mm}]$
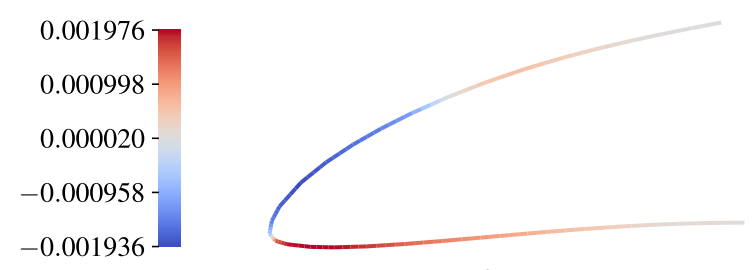

(b) Sensitivity w.r.t. torque $\frac{\partial e}{\partial M_{t}}[\mathrm{~mm} / \mathrm{N} \cdot \mathrm{mm}]$

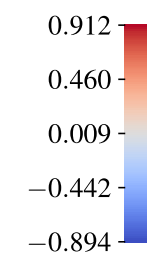

(d) Sensitivity w.r.t. actuation point in $y \frac{\partial e}{\partial y_{a}}[\mathrm{~mm} / \mathrm{mm}]$

$$
\begin{array}{r}
0.0002766 \\
0.0001369- \\
-0.0000028- \\
-0.0001425- \\
-0.0002823
\end{array}
$$

(e) Sensitivity w.r.t. Young's modulus $\frac{\partial e}{\partial E}[\mathrm{~mm} / \mathrm{MPa}]$

Figure 9: Design sensitivities of the error $e$ in morphed configuration 
Table 1: Design sensitivities of the maximum stress $\sigma_{\max }$ and the root-mean-square error $e_{\text {rms }}$

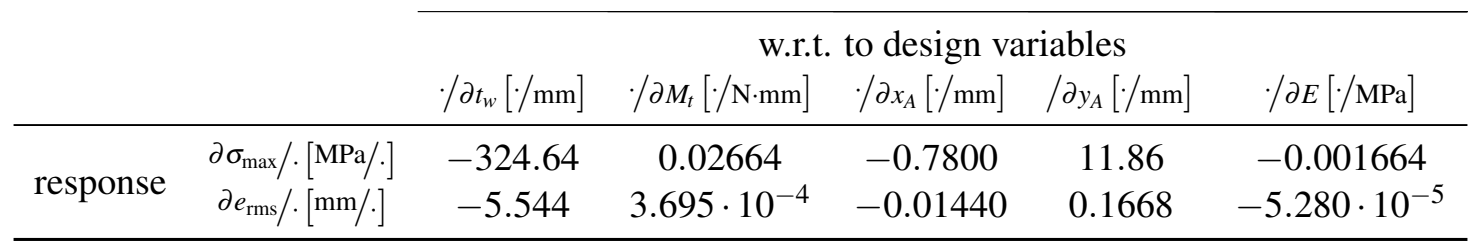

Table 2: Computational effort of semi-analytical and numerical sensitivity analysis

\begin{tabular}{cc}
\hline method & computation time $[\mathrm{min}: \mathrm{s}]$ \\
\hline semi-analytical sensitivity analysis & $03: 32$ \\
numerical sensitivity analysis & $14: 48$ \\
\hline
\end{tabular}

comparison shows the high efficiency of the implemented semi-analytical sensitivity approach. The computational effort of the numerical sensitivity analysis is given by the sum of $n_{\mathrm{x}}+1$ primal evaluations and is given here by 6 evaluations with a mean evaluation time of $02: 28$ [ $\mathrm{min}: \mathrm{s}$ ]. The computational effort of a system evaluation with the semi-analytical sensitivity analysis is less then twice $(1.43 \times)$ the computational effort of one primal evaluation due to the simplification shown in $\S 3.3$.

\section{CONCLUSION}

The present work introduces an efficient sensitivity analysis of flexible multibody dynamics with a semi-analytic direct differentiation approach of generalized- $\alpha$ time integration with NewtonRaphson iterations for a morphing wing concept. This concept for a morphing wing leading edge with a trailing flap for a high-speed and a low-speed configuration increases the performance in the wide speed range of a high-performance sailplane. The morphing wing leading edge models the external wing skin with flexible elements, while the actuation mechanism is modeled with rigid bodies. Special interest was devoted to the deviation error from the target shape in morphed configuration and the stress values in the wing skin in addition to both of these sensitivities. Semi-analytical sensitivity analysis is utilized for its computational efficiency and accuracy. The calculated design sensitivities include those with respect to geometric properties, material properties and the position and torque of the actuator. This sensitivity analysis is the basis for future uncertainty analysis and gradient-based design optimization of the morphing wing and its actuation mechanism. The maximum stress is considered as material limit, though the consideration of the fatigue (including sensitivities) would be of great practical use. This work is an integral part of the integration of a design optimization framework to optimally design the actuation mechanism and geometry of a morphing wing leading edge.

\section{ACKNOWLEDGMENTS}

This work is supported by the project CRC 2017 TN2091 doloMULTI Design of Lightweight Optimized structures and systems under MULTIdisciplinary considerations through integration of MULTIbody dynamics in a MULTIphysics framework funded by the Free University of BozenBolzano. Further support was provided in the framework MILAN - Morphing wIngs for saiLplANes, funded by the German Federal Ministry for Economic Affairs and Energy under the grant of the German Federal Aviation Research Program (Luftfahrtforschungsprogramm, LuFo) V-3. 


\section{REFERENCES}

[1] Achleitner, J., Rohde-Brandenburger, K., Rogalla von Bieberstein, P., Sturm, F., Hornung, M.: Aerodynamic design of a morphing wing sailplane. In: AIAA Aviation 2019 Forum, Reston, Virginia, American Institute of Aeronautics and Astronautics (2019)

[2] Sinapius, M., Monner, H.P., Kintscher, M., Riemenschneider, J.: DLR's morphing wing activities within the European network. Procedia IUTAM 10 (2014) 416-426

[3] Sturm, F., Achleitner, J., Jocham, K., Hornung, M.: Studies of anisotropic wing shell concepts for a sailplane with a morphing forward wing section. In: AIAA Aviation 2019 Forum, Reston, Virginia, American Institute of Aeronautics and Astronautics (2019)

[4] Rudenko, A., Hannig, A., Monner, H.P., Horst, P.: Extremely deformable morphing leading edge: Optimization, design and structural testing. Journal of Intelligent Material Systems and Structures 29(5) (2017) 764-773

[5] Reinisch, J., Wehrle, E., Achleitner, J.: Multiresolution topology optimization of largedeformation path-generation compliant mechanisms with stress constraints. Applied Sciences 11(6)(2479) (2021)

[6] Gufler, V.: Multibody dynamics and optimal design of a Tyrolean weir cleaning mechanism. Master thesis, Free University of Bozen-Bolzano (2019) Advisors: E. J. Wehrle, R. Vidoni.

[7] Gufler, V., Wehrle, E., Vidoni, R.: Multiphysical design optimization of multibody systems: Application to a Tyrolean weir cleaning mechanism. In: Advances in Italian Mechanism Science. Springer International Publishing (2021) 459-467

[8] Wehrle, E., Gufler, V.: Lightweight engineering design of nonlinear dynamic systems with gradient-based structural design optimization. In: Proceedings of the Munich Symposium on Lightweight Design 2020. Springer Berlin Heidelberg (2021) 44-57

[9] Gufler, V., Wehrle, E., Zwölfer, A.: A review of flexible multibody dynamics for gradientbased design optimization. (submitted)

[10] Shabana, A.A.: Dynamics of multibody systems. 5 edn. Cambridge University Press (2020)

[11] Chung, J., Hulbert, G.: A time integration algorithm for structural dynamics with improved numerical dissipation: The generalized- $\alpha$ method. Journal of Applied Mechanics 60 (1993) $371-375$

[12] Wehrle, E., Gufler, V.: Analytical sensitivity analysis of dynamic problems with direct differentiation of generalized- $\alpha$ time integration. (submitted)

[13] Achleitner, J., Rohde-Brandenburger, K., Hornung, M.: Airfoil optimization with CST parameterization for (un-)conventional demands. In: XXXIV OSTIV Congress. (2018) 117120

[14] Bongers, J.: Implementation of a new transition prediction method in XFOIL. Masters thesis, TU Delft, Netherlands (2008)

[15] Drela, M.: XFOIL: An analysis and design system for low Reynolds number airfoils. In: Low Reynolds Number Aerodynamics, Lecture Notes in Engineering. Springer Berlin Heidelberg (1989) $1-12$

[16] Kreisselmeier, G., Steinhauser, R.: Systematic control design by optimizing a vector performance index. In: International Federation of Active Controls Symposium on ComputerAided Design of Control Systems. Volume 12. Elsevier (1979) 113-117 